\title{
Functional anatomy of pelvic floor
}

\author{
Salvatore Rocca Rossetti \\ Professor of Urology, University of Torino, Torino, Italy.
}

\begin{abstract}
Summary Generally, descriptions of the pelvic floor are discordant, since its complex structures and the complexity of pathological disorders of such structures; commonly the descriptions are sectorial, concerning muscles, fascial developments, ligaments and so on. On the contrary to understand completely nature and function of the pelvic floor it is necessary to study it in the most unitary view and in the most global aspect, considering embriology, philogenesy, anthropologic development and its multiple activities others than urological, gynaecological and intestinal ones.

Recent acquirements succeeded in clarifying many aspects of pelvic floor activity, whose musculature has been investigated through electromyography, sonography, magnetic resonance, histology, histochemistry, molecular research. Utilizing recent research concerning not only urinary and gynecologic aspects but also those regarding statics and dynamics of pelvis and its floor, it is now possible to study this important body part as a unit; that means to consider it in the whole body economy to which maintaining upright position, walking and behavior or physical conduct do not share less than urinary, genital, and intestinal functions. It is today possible to consider the pelvic floor as a musclefascial unit with synergic and antagonistic activity of muscular bundles, among them more or less interlaced, with multiple functions and not only the function of pelvic cup closure.
\end{abstract}

KEY WORDS: Pelvic floor; Anatomy; Function; Micturition; Continence; Defecation; Coitus; Posture.

Submitted 17 October; Accepted 31 December 2015

\section{INTRODUCTION}

Generally the descriptions of the pelvic floor are discordant, since its complex structures and the complexity of pathological disorders of such structures; commonly the descriptions are sectorial, concerning muscles, fascial developments, ligaments and so on. On the contrary to understand completely nature and function of the pelvic floor it is necessary to study it in the most unitary view, in the most global aspect, considering embriology, philogenesy, anthropologic development and its multiple activities others than urological, gynaecological and intestinal. The pelvic floor function is mainly involved in postural aptitude, in the upright position, in the gait, in the physical conduct. Only considering those aspects all together it is possible to have a clear view of pelvic floor anatomy and function. Although urologists, gynecologists and gastroenterologists generally consider the pelvic floor only for the support activity of pelvic organs and for their functions, it is necessary not to neglect its great contribution for the behavior life to appreciate its value in the body economy; on the other hand also the pelvic organs function is part of the behavior life. This one is greatly based on pose and on body movements as well as on some functions to be done at the right time and at the right place; in that the unitarity of pelvic floor functional value is recognizable, because pelvic floor function, gait, and all movements of behavior life are functionally linked.

So it is worthwhile to consider some notions that very few have to do with urology "sensu stricto" but that help to understand the true value of the pelvic floor: Sherrington (1) underlined "the posture follows movements like a shadow", that means that posture is the evolutional results in which every living beings must adapt themselves to the environment in which they live, where they can carry out their static and dynamic activity. According to a strictly anthropologic point of view it was affirmed that we became humans in our loins and in our pelvis well before than in our brain (2), because pelvic modifications made possible the constant upright position, superior limbs freeing, exclusively biped gait and human beings delivery. Today the personality development and the body structure are believed to proceed simultaneously and then the habitual posture (how to show oneself) express the personal character that one "represents how to stay in the world, to face the reality, that means the visible conclusion of all that allowed us to outlive" (3). Today psychological research studies the relations between posture and personality and consider the intelligence (capacity in solving problems) like a body dimension, the mind like hands and implements generally used extension. So for all that there are evidences on the necessity to consider the pelvic floor in the must unitary shape and not only in relation to urinary, gynecologic, and gastrointestinal functions.

\section{Generality}

To start, it must be remembered that this part of the trunk, particularly the pelvis or bony basin, has been built in the evolution for organs containment and protection; our axial skeleton, that means vertebral column and pelvic bone represents the fish vertebra-type residue 
(it is well known that we came from sea), that posteriorly covers the spinal cord and anteriorly the visceral organs like a bony or cartilaginous carapace. Such a disposition in human body is identifiable only on chest, but the nature proceeds from simple to complex, living in the complex the simple trace (of what is found in lower in zoological scale animals), the mylohyoid line, the hyoid bone, the sternum xipoid process, the abdominal alba line just depict the traces; moreover sometimes on these structures it is possible to find some ossifications testifying the old carapace trace. Obviously the pelvic floor cannot be consider independently from the bony pelvis, whom evolution greatly was owed to the upright position, the biped gait and the skull capability increasing, which allowed the shortening of iliac wings, the increasing of their width and superior and inferior straits width, the pelvis verticalization, the sacrum posterioralization with consequent lumbar lordosis, dorsal kifosis, cervical lordosis and occipital hole anterioralization; and also the lateralization of the hip cavity and the femur axis. The upright position determined the center of gravity elevation at the fifth lumbar vertebra peduncles; from them the force lines coming down from top diverge laterally at each side towards the iliac-sacral joints and discharge the head and trunk weight on the hip joints and on inferior limbs; as many force lines from ground go up towards the pelvis bone lengthways the femur axis; this one, since the pelvis enlargement and the consequent hip joints lateralization, breaks of at level of the femur neck and bends itself internally and posteriorly; so these force lines make a compression action on the pubic rami, necessary for opposing to the lateral expansion forces due to the new skull dimensions and to the changed delivery mechanism (clear results of that compression is visible on malacic pelvis like in rickets or osteoporotic bones: $\mathrm{Y}$ pelvis deformation, since the push from femurs is stronger that the pubic arch resistance) (4).

For our study it is convenient to consider the body standing up, in vertical position (not supine or in lithotomic position), as well as the classic anatomic position for which it is considered the meaning of anterior, posterior, medial, lateral and so on. In the vertical position the anterior-superior iliac spine and pubis are on the same coronal plane, the pelvis opening looks anteriorly and the ischio-pubic branches are parallel to ground as well as the urinary, gynecologic and intestinal hiatus; in that position the abdominal and pelvic visceral pressure falls on pelvic bones and is minimized on the muscular framework.

The bony pelvis has a funnel shape with the larger part on the top (plane passing for the iliac crests) and the narrow one below (plane passing for the ischial tuberosities and coccyx apex); iliac wings and fossae are topographically part of the abdomen, lodging abdominal organs surgically attackable; in this way only the part included between superior strait (plane from pubis to sacral promontory through the "innominate" line) and inferior one (plain from ischial tuberosities to coccyx apex) is considered as pelvis; perineum is the pelvic bottom closure. For all those considerations, it happens that abdominal organs have no place to be lodged below, so they move upward together with the peritoneum from which they are covered or received; consequently below the peritoneum a large connectival space: called subperitoneal space is formed. Two diaphragms enclose all the pelvic-abdominal organs: superiorly the thoraco-abdominal diaphragm or diaphragm properly called, inferiorly the pelvic diaphragm; both have an intricate structure and embriologic derivation (5-7).

The pelvis is partially closed inferiorly from a series of muscles, fasciae, ligaments for the better understanding of which it is necessary to remember their embriologic and philogenetic derivation (8). In concomitance with the first outlines of pelvic organs formation, two separate groups of muscles start to grow: tail muscle or pubo-caudal muscle and cloacal or Gegenbauer muscle; from the former the ischio-coccigeus muscle, levator ani muscle and pubo-sacral and sacro-spinal ligaments will descent, from the latter the sphincters. After the genito-urinary septum will separate the rectum posteriorly from the urethra-vagina anteriorly, the Gegenbauer's muscle will divide itself in two sphincters, one for the urethra and one for the rectum; successively ischio-cavernous muscle, bulbo-cavernous muscle and superficial and deep transverse muscles are added; the sacro-touberous and sacro-spinous ligaments are a kind of fibrous transformation of caudal muscles.

Already in fetus period (9) and much more in adult one those muscles or caudal and cloacal groups of muscles are puzzling among them, in sense that they don't show themselves separate, as in some marauders (tiger); the puzzling or mixing of muscles of different derivation, function and innervation (sacral plexus the former, pudendal plexus the latter) is the main reason of the contrasting descriptions of muscles and pelvic ligaments in men. For instance, if we consider the mixing and sometimes the fusion of levator ani fibers with the deep transverse muscle and with the urethral sphincter where this one surrounds partially the prostate we find the reason of difficulty in understanding the true function of each group of pelvic muscles; apparently the upright posture and the bipede gait, compared to quadrupedes, are almost part of the reason of above mentioned muscular mixing.

The pelvis bone above cited modifications, due to the new behavioral conditions, have caused the functional unitariety among the musculo-fascial structures of pelvis cavity (small pelvis) in a concordant function of support and activity of pelvic organs (bladder, urethra. womb, vagina, rectum, anus). Muscles of such musculo-fascial structures shrivel, relax, synergize, antagonize, stabilize (stabilizer muscle is that one which shrivel isometrically to keep stable the body part towards which the movement goes).according with pelvic organs activity (filling, voiding) or resting; their fibers are for $2 / 3$ of the first type, red fibers at slow contraction and for $1 / 3$ of the second type at quick contraction $(10,15)$. Their activity doesn't work out only for visceral functions, but also for supporting organs, for keeping the correct position and, as we said, for maintaining the posture, the deambulation and correlate problems as respiration (12); so synergism and antagonism happen also with other muscular groups of different part of the body.

Considering the framework of this part it is easy to 
understand synergism and antagonism among pelvic muscles and those of the abdominal anterior part. The pelvic diaphragm, pubo-coccigeous, ileo-coccigeous, and ischio-coccigeous (levator ani) puts itself like an hammock of pelvic and abdominal elastic containment; the transverse abdominis muscle being the deeper is the most synergic with pubo-coccigeus and ischio-coccigeus; also the gluteus major solidarizes with pelvic musculature to maintain the upright position and posture.

The pelvic musculature supplies a fundamental contribution to stability and body movements (many of posterior thigh muscles, particularly abductors, take insertions on ischiatic tuberosity and on sacro-tuberous ligament); the solidarity of pelvi-trochanteric muscles, particularly internal obturator and piriform and rotators of thigh, is also very important; their fasciae assure solidity to the pelvis framework (13).

Commonly but wrongly the pelvic floor muscles activity is considered in the same manner of majority skeletal muscles activity as moving (one or more) skeletal bones in a coordinated contraction or decontraction of agonists, antagonists, stabilizers; but that is wrong because among the pelvis bones only coccyx is partially movable. It is well known that the muscular contraction is either isotonic or isometric; this is true and evident for monoor poly-joints muscles, whose contraction creates the displacement of a skeletal segment, as forearm flection on the arm, like a lever with fulcrum on elbow; the isometric contraction doesn't create any displacement thanks to the antagonists action; that nevertheless also functions for skeletal displacement in regulating its degree, amplitude and rapidity. Muscles of pelvic diaphragm, except for pubo-rectalis (visceral ones), insert themselves on fixed bony segments, such pubis, ilium, ischium and sacrum; the slightest movements, nutation and contra-nutation, of the latter happen only in the expulsive period of delivery $(14,15)$; the coccyx, scarcely movable in the same phase is generally kept fix by the major gluteus muscle tone and contraction. The importance of those muscles tonic phase and the characteristics of their contraction have to be considered.

The pelvic muscles activity is essential in keeping a constant although variable tonic condition and in contracting in certain circumstances like urination, defecation, coitus. These muscles tone is quite peculiar, consisting in an intermediate stage between tone and contraction and since it is constant, or in any case prolonged, it would be exposed to exhaustion (typical of striated muscle contraction) if a series of sophisticate neurological interactions wouldn't avoid it.

The reason why this musculature is different to the others concerning tone lies also in the peculiar shape of bony pelvis and in the obliged position of the organs since the narrowing of the pelvis lower part (3).

For descriptive convenience, it is used to consider in the pelvis closure a superior strait, pelvis diaphragm, a medium one, uro-genital diaphragm and an inferior one, perineum; such a distinction is completely artificial and only partially responds to systematic anatomy requirements in muscles, fasciae, vessels and nerves description. That being stated, I think not to do without in respecting the distinction because it is still kept in the uro-gynecological literature.

\section{Pelvic diaphragm}

Pelvic cup closure, since its funnel conformation, is insured by the musculo-fascial solidarity whose fibrous and muscular structure are steady attached to bony prominences, which were spirally developed starting from the primitive nucleus of cotyl from which ilium, ischium and pubis originated (8). Generally it is said that the pelvic cup is occupied by ischio-coccigeus and elevator ani muscles; the latter formed by ilio-coccigeus and pubo-coccigeus bellies; in that matter pubo-, ilio- and ischio-coccigeus form the pelvic diaphragm, with their insertions on ilium, ischium an pubis.

In recent literature there are not few divergences regarding the description before related, that, strictly anatomically speaking, I think is correct. Indeed there are Authors who consider. the levator ani the only muscle of pelvic diaphragm (31), that means they include the ischio-coccigeus as part of levator ani. Oppositely other Authors consider the coccigeus the only muscle of pelvic diaphragm with its pubic, iliac and ischiatic insertions. Some but not few Authors use to separate bellies for support, ilio-coccigeus, ischio-coccigeus and a little part of pubo-coccigeus, from those for visceral function, puborectalis, which is the bigger part of pubo-coccigeus (in its turn, part of levator ani). Incidentally little internal fibers of pubo-rectalis are the only part of levator ani which really lifts anus, since their insertions on anal subcutaneous. Evidently those descriptive divergences certainly don't help for the better understanding of the subject; so necessity was felt to apply at international nomenclatures (18). The anatomic nomenclature gives names to muscles on the base of shape (deltoid), of bellies composition (biceps), of insertions (sterno-cleido-mastoideous), or of function characteristics (supinator); so I don't think it is scandalous in considering the pelvic musculature on the base of its function, at least in distinguishing support from visceral function. If we consider that biggest part of levator ani and ischio-coccigeus attend to organ support and to the pelvic cup closure, while only pubo-rectalis bellies of levator ani reach rectum, urethra and vagina, call the latter viscerals on the base of function could seem logical. Since pelvic disorders are prevalent in women there are Authors who studying those muscles only for their uro-gynaecological functions, use to call them pubo-urethral, pubo-vaginal, pubo-perineal, puboanal on the base of an excessive functional nomenclature. All that didn't contribute to clarity about $(20,28)$.

Calling pelvic muscles according to support or to visceral function, we may consider the pelvic diaphragm made by the levator ani whose bellies, ilio, ischio and pubococcigeus have an exclusively bony insertion and constitute the plate and the pelvic excavation closure, while the internal bellies of pubococcigeus practice a visceral action; those are frequently called pubo-rectalis; since their action is made not only on rectum, but also on urethra and vagina, it is better to call them pubo-visceralis. The former are put between bone and bone and exert tone and isometric contraction; the latter.have an isoton- 
ic contraction and don't insert themselves directly and properly on organs but with their contraction they shorten and make a leave action as fulcrum followed by a movement of the organ or of a part of it. All of them contribute to circumscribe the hole through which prostate, urethra, vagina and rectum pass; these are kept in place thanks to a connectival thickness which makes connection between organ and muscle; some Authors call that connective tissue hiatal ligament (30).

The support muscular bundles insert themselves directly or through fascial coverings on perineal tendineous center, the perineal body, on which the three perineal fasciae converge together with cloacal and caudal muscles in an anatomically and functionally unitary constitution. The pubo-visceral bundles (part of pubo-coccigeous) have a quite oblique direction and go down to encompass posteriorly the rectum (pubo-rectals properly said) and form something like a hammock at recto-anal level; they also encompass posteriorly urethra and vagina in women, prostate and urethra in men; they adhere to and partially joint themselves with the urethral striped sphincter and make like a ring all around vagina (28).

It is fundamental for function understanding to consider the muscular fibers direction and their force lines; in the pelvic diaphragm that direction is grossly sagittal, but their true action must be considered on the base of their insertions or fusions with pelvic fascia, ligaments, tendons and so on.

Differently to what dissection anatomy shows the pelvic musculature being funnel like up to down, magnetic resonance, that means living anatomy, demonstrates the disposition of their bundles be horizontal, as a plate or flat console and not like the cup walls (pubo-visceral bundles are the exception). This divergence is due to the complete absence of muscles contraction in cadavers while in living beings a firm tone is constantly present, which expires only during micturition and defecation (on the recto-anal hammock tone perseverance still are some divergences in literature).

The tri-dimensional study of levator ani (20) shows that the anterior portion, pubo-visceral, is made as we said, by bundles downward obliquely directed; they partially insert themselves on perineal body ( tendineous center of perineum) and in bigger part they make a sling posteriorly to rectum, vagina and urethra; their contraction tighten the uro-genital diaphragm hole pushing rectum, vagina and urethra towards the pubic bone. The posterior part of levator ani, ilio-coccigeus, ischio-coccigeus and some bundles of pubo-coccigeus are put horizontally, in a flat disposition, forming the pelvic organs support; they become oblique, funnel like, in case of direct or neurological lesions with consequent prolapse risk.

The muscular and fascial fibers direction explains their action effect, particularly well studied in women (28); the force lines developed in median perineal aponeurosis and in deep transverse of perineum muscle (often hypotrophic or absent in women (23) are directed towards ischio-pubic branches and reinforce the urethra and vagina closure action. Since muscles contraction can pull, but not push (15) synergism and antagonism must be respected also for pelvic muscles in their relations with pelvi-trochanteric muscles (obturator and piriform) and with thigh muscles, particularly with adductors (adductor, gracile, pectineous); that is worth especially for mono- or bi- foot-lever movements (4) and also for the help that those muscles contraction could give during movements for which urinary and fecal continence needs the greatest effort.

As already said, the pelvic cup closure is done by a strong musculo-aponeurotic framework, for that the fascial and aponeurotic structures are of an anatomic and functional importance; they in certain sense precede origin and organization of muscles bundles, almost for what is concerning the mesenchimal, sub-mesothelial and vascular derivation $(8,24)$. To have a quick idea of that it is well worth to consider that the bony pelvis funnel-like shape forces the organs with their peritoneum to go up towards the abdomen since they have no place in the narrow strait below; for that a conspicuous subperitoneal connectival tissue is formed, called endopelvic fascia; this one, guided by zonal vessels already present in that district, became divided in lodges for the organs; thus from hypogasric artery and its prolongation (anteriorly obturator artery, posteriorly lateral sacral artery) hypogastric sheath is formed which connects pubis and sacrum; in the same time in coronal plane other bands or sheaths are formed; going from forward to backward umbelicoprevescical sheath is strechted between the two unbelical arteries; the vescico-rectal sheath between the two vesciculo-deferential arteries; the Denonvilliers sheath between the two median rectals arteries (some Authors improperly compare this sheath with the women large ligament; the true equivalent otherwise is the recto-vaginal sheath); in this manner retropubic space, vesico-prostatic lodge, utero-ovaric lodge, rectal lodge and presacral space are delimited (30).

The hypogastric fascia thickens, as said, in the pubosacral ligament and extends covering all pelvic cup muscles (particularly internal obturator and levator ani) in the pelvic sheath formation; this one is also called deep perineal sheath or superior to be distinct from the inferior or superficial and from median one; between levator ani and internal obturator the pelvic fascia thickens forming the so called white line or levator ani tendineous arch which joints pubis and ischiatic spine and on which some fibers of elevator insert. Each pelvic diaphragm muscle has its perimysium, more or less thin, sticked with pelvic fascia, which wraps up also the levator inferior side in what some Authors call.

improperly endopelvic fascia; as we said the endopelvic fascia is properly the sheath put between the visceral peritoneum and pelvic fascia that is particularly thick in correspondence of cardinal ligaments and paracolpium. It is well known that pelvic organs, where not covered by peritoneum, have a more or less thin adventitia which is in continuation with bands or zonal ligaments. Therefore urethra, prostate, bladder neck, base and side, vagina, womb neck and rectum are sagittally adherent to pubosacral ligament through bands especially well visible at urethral and vaginal level; the connections with pubovisceral muscles (internal part of pubo-coccigeous, also called pubo-rectalis) superimpose on them. These connections are generally connectival in nature, like adventitia and perimysium; the only exception is the adhesion 
of the internal part of pubo-rectalis muscle whose fibers scatter on prostatic capsule; some Authors call that adhesion "prostate levator muscle" (30).

The adhesion and connection among organs, fasciae, ligaments and muscles connective tissue is more or less dense, elastic and plentiful in conformity with organs, sex, and zones, what is not without importance for continence and micturition mechanisms; this connective mass represents a true and proper structure (pubo-vesical urethral vaginal) that "joints" these organs to pubis, pelvic fascia and tendineous levator arch; the structure is commonly considered to be part of pubo-urethral ligaments which are the first part of pubo-sacral long ligament. This dense connectival structure seems to play an important role in the vagina and urethra compression against pubis together with the well-known musculo-fascial structures in maintaining the continence threatened by intra-abdominal sudden increased pressure (28).

As it is a functionally important synergism between pelvic muscles and those of trunk and limbs it exists a not less important synergism among fasciae, ligaments, and muscular structures of the zone.

The fasciae are formed by collagen, elastic tissue and smooth muscles; they also have proprioceptors which are activated by the ligaments stretching, which by reflex induces the muscular contraction useful to abolish ligaments and fascial tension; so it exists a musculo-fascial and ligaments balance that gives the correct grade of tone and contraction of pelvic floor (32).

Evidently the muscular and aponeurotic organization was obliged to adapt itself to pelvic organs presence and functioning and in the same time to the necessity in ensuring to the pelvis the upright position stabilization and that one of movements on one or both feet. This task is obtained by levator ani with its pubo-visceral bundles and by coccigeus with pubic, iliac and ischiatic derivation bundles; practically all the other bellies of levator ani (indeed, as already said, some Authors assimilate ischio-coccigeus muscle to levator ani) (23).

\section{URO-GENITAL DIAPHGRAM - PERINEUM}

Below the pelvic diaphragm, made by levator ani, the urogenital diaphragm lies formed by perineal membrane (or midperineal aponeurosis) which spans the deep perineal muscles (or deep transverse muscles); some Authors (16) believe that for the urogenital one the word diaphragm is improper; truly there isn't a real and proper separation ( this indeed is the meaning of diaphragm) but a strengthening which joints the pelvic diaphragm to the underlying perineum; its anterior part lets urogenital conduits pass to which it is in certain manner connected.. The urogenital diaphragm or triangle is formed, as said, by midperineal aponeurosis or Carcassonne's fascia and by deep transverse perineal muscle; the unitariety of pelvic floor is well visible in this strong aponeurosis disposition and structure, strained between the two ischiopubic branches; its apex looks anteriorly toward the pubic symphysis; this triangle, bored by urethra and vagina, fills the space where in the anterior part of perineum the two levators ani don't joint each other and it is put both anteriorly and posteriorly to urethra and vagina; the pre- urethral part, also called transverse perineal ligament is strongly connected to ischiopubic branches and to obturator fascia; it has very dense structure so much to become so sharp to guillottine the urethra in the ischiopubic fractures dislocations. The retro-urethral part divides itself in two sheets, inferior, well thick and pressure-proof, and superior thin; between them Gouthrie or deep perineal muscle lies; this is quite thin in women, even if existent. The two sheets of perineal membrane are in continuation: the inferior one with the inferior perineal fascia covering the superficial transverse perineal muscle; the superior one with Denonvilliers fascia or the levator perimysium; from that it seems clear that the urogenital triangle and the whole perineal framework are compact and tightly linked to the overlanding pelvic diaphragm and to the underline ischio- and bulbo-cavernous muscles.

Although generally the pelvic cup closure is described as tree superimposed layers, pelvic diaphragm, urogenital diaphragm and perineum, really, as we said, this division results quite artificial at least for the two presumed inferior layers; indeed it is impossible not to include the urogenital diaphragm in the perineum and not to include in this one descending levator pubo-rectalis bundles, belongings to pelvic diaphragm; in this connection there are Authors who consider perineum the musculo-fascial whole which represents the outlet pelvis closure; so it is clear why the international literature rather has to speak generically of pelvic floor, also keeping dictions like pelvic diaphragm, urogenital diaphragm and perineum. With the aim to recall literature names or dictions it is good to remember that the deep perineal transverse muscle part included between the two Carcassonne's sheets is also called Guthrie muscle; this one is actually the retro-urethral portion of perineal deep muscle; Wilson muscle is instead the whole of deep perineal muscle fibers which scatter on superior Carcassonne's sheath and adhere at external urethral sphincter; this one is a true circular muff around the urethra just below perineal membrane; on top those fibers disperse themselves round the prostate without joining posteriorly; at this level some muscular fibers joining prostate, levator ani and longitudinal rectal bundle are called recto-urethral or Henle muscle (varies structures synonyms were cited with the aim to facilitate for readers the comprehension of those differently called in literature).

Genitourinary hiatus in women is obviously bigger because of the vagina presence between urethra and rectum, the deep perineal muscle hypotrophy and the presence of pubo-urethral fibers, compressor vaginae, which are the internal part of levator ani, also called pubo-visceralis.

The perineum also lodges in addiction to superficial and deep (transverse) perineal muscles, striated urethral and anal sphincters and bulbo and ischio-cavernous muscles in both sexes; The levator ani bundles called properly pubo-visceral because they join urethra, vagina and rectum go down in perineum making a hammock-like sling round the recto-anal junction where part of them melt with the longitudinal bundle of rectum and with striated anal sphincter; the more superficial of those fibers reach the anal subcutaneous and consequentially they are the only ones deserving the name levator ani. As we said the 
pubo-visceral fibers are often called pubo-rectal. Among varies contradictions of this topic there are some concerning the real outline and function of pubo-rectal muscle; there are Authors (33) who through dissection and microscopic research deny that this muscle belongs to levator ani to which they only ascribe pubo-coccigeous and ilio-coccigeous bellies; so pubo-rectal muscle would be part of external ani sphincter (sphincteric complex) thanks to the confluence of its fibers with that muscle and with the longitudinal rectal fibers; the two levators of right and left side would cross each other at the anococcygeus raphe on which they don't put insertion. The pubo-visceral most anterior portion makes an analogous loop around the mid urethra part and vagina, in women creating the so called compressor vaginae muscle.

Urethra, vagina and rectum are kept in place and in certain sense supported by pubosacral ligaments to which they adhere through connections called from time to time pubo-urethral, pubo-vaginal, pubo-rectal, sacrorectal ligaments. The pubo-sacral ligament is in continuity with the pelvic fascia of which on the other hand represents a thickening of what is the many times mentioned fascial or better musculo-fascial unitariness.

This is a synthetic view of the anatomical region and its musculo-aponeurotic structures settlement; to us it isn't to be surprised from the strictly surgical habit in emphasizing fragments of fasciae or ligaments variously called in the aim to show their positive or negative value in the interpretation of pelvic dysfunctions as incontinence or ptosis, and consequent surgical operations suggestion.

\section{Continence-micturition}

Micturition is the abolishing of continence and the outside expulsion of urine. The muscular mechanisms of this function are well known: inhibition of cervico-urethral contraction, detrusor contraction and if necessary anterior abdominal musculature contraction (to which micturition, defecation, vomiting and delivery is classically tribute). For what is concerning the pelvic floor, sphincteric inhibition a part, the micturition needs pelvic diaphragm musculature decontraction (chiefly pubo-coccygeus muscle); a sequence of sophisticated neurologic, medullar and encephalic mechanisms rules the coordination among orthosympathic inhibition (cervical trigone relaxation), parasympathic action (concentric detrusor contraction) and levator ani tone abolishing; the coordination is essential to obtain the sphincteric and pelvic relaxation just before the bladder contraction (33) and also to respect the fact that those phenomenona are subjected to will and subconsciousness to be carry on where and when is convenient. A series of very interesting and particularly complicate reflexes rules all that $(11,35)$. Other reflexes are ruled by superior neurologic centers concerning pelvic floor tone and contraction in a constant adaptation to sudden body balance variations and its consequent micturition possibility. Obviously the function of this reflexes acts through inhibition (quantitatively bigger impulses) and muscular action; the result of this one on starting and maintaining the micturition, for what is concerning the pelvic floor support, is obtained through the total or partial relaxation of its muscles with the consequence not only of the urethra going down but also of its shortening; lenght decrease is useful for the micturition because, according with Laplace law length decrease create wall resistance decease (in wall distensible tubes, like vessels or hole organs the filling up deforms the wall, which develops a tension compared to the lengthening); obviously the shortening of the urethra causes the decrease of resistance (33).

Considering the closure and opening of bladder and urethral neck it is to remember hormonal effect, particularly that of catecholamines: for instance it is known that passing from supine to upright position a sudden increase of catecholamines in blood creates a pelvic floor tone and contraction increase with consequent continence mechanism strengthening; that is pathologically evident in case of long standing hypertone with the impossibility to pass urine (patients who mast pass water lying); in some spinal cord lesions the denervation of smooth urethral muscles creates a bigger sensitivity to catecholamines (decentralization supersensitivity) with pelvic floor hypertone. In opposite side there are cases, fortunately rare, in which the global pelvic floor flaccidity causes the posterior prolapse of the bladder and big urethral bend on perineal body with micturition impossibility (35).

For what is concerning the pelvic floor relaxation during normal micturition it must be considered that the relaxation happens only for the pubo-visceral bundles and not for all those of levator ani; Shafik (33) asserts that the increasing tone or really the support levator bundles contraction causes the bladder neck and urethra relaxation; such effect would be reflected on micturition mechanism thanks to the Poiseuille's law regarding resistance to flow modifications (also this low was studied for blood vessels relaxation). According to that research the pubo-rectal muscle would be contracturant and at opposite the levator ani relaxant at sphincteric level; practically the pubo-urethralis relaxes freeing the urethra, the levator contracts making traction on urethral walls, obtaining the opening.

If I may open a parenthesis in appendix of this little chapter concerning the physics laws in the urinary phenomenona interpretation, I would like to remember here what in the theatre I showed to students and fellows during radical cystectomy; I let to put the removed piece on a rigid place and pushing hardly on the bladder I showed that no drops of urine got out; I utilized that show to remember them that the passive or physic continence can be done only from active muscular force to let urine pass; I also remembered that some urologists (erroneously) call upon Pascal's law to demonstrate that, being the closed bladder neck radius equal zero it could be necessary an infinitive force from the bladder to open it; bladder neck and urethra are them opened thanks to a muscular activity for starting and maintaining the micturition (37).

In the last few years research appeared in literature regarding urinary continence, meanly with the aim to repair its dysfunctions; the research where particularly concerning spontaneous incontinence, then female incontinence.

Sonography, Magnetic Resonance, Functional Magnetic Resonance regarding encephalic areas for movements and 
stress, Microscopy, Histology, Histochemistry, Molecular research for receptors, Electromyography have greatly increased our knowledge of the topic. For what is concerning the pelvic floor muscle-aponeurotic structure active in continence mechanism, de Lancey (38) anatomical and functional systematization is to well known to be here recalled. It seems instead useful to describe some research that doesn't agree completely with that thesis; for instance Sasaki and al. (39) through meticulous histologic and histo-chemical research believe that connective-elastic and smooth muscles transition has a particular rule in "fibrous perineal skeleton" constitution. This one contributes to urinary and rectal continence in women through fusion and synergic action of the abundant perineal smooth muscle (urethra, rectum, vagina) with levator striated one; the Authors describe at each side of urethra and vagina a smooth muscular mass in a catamaran ship configuration whom seal would be represented by rectal-vaginal fascia; the two lateral masses are made from urethra and rectal smooth sphincters, longitudinal intrinsic rectal fibers and vaginal adventitia; to those the fibers of striated rectal sphincter and levator ani unify and melt. The pubo-vaginal muscle action represents the active continence value of "fibrous skeleton" but concerning the ptosis it is quite independent.

An other divergent aspect compared with Ashton Miller and de Lancey theory is that regarding on which part of the urethra the abdominal pressure and the pubo-visceral muscle action exert their power (40); also in past time frequent debates happened regarding Enhorming G. theory (42) on pressure percentile fall on the abdominal portion of the urethra compared to that one on the bladder $(50 \%$ according to some, $75 \%$ to others; see discussion in 35, pages 734-35) and regarding the urethral bend on perineal body. Recent interesting studies still confirm those discrepances (41) and confirm the existence of cases in which normal continence was present also when all portions of urethra laid below the perineal body (in contrast of Enhorming theory). With regard of this subject Lazarswski MB (40) speaking about stress incontinence biomechanics shows its theory on the action of so called non-permanent structures on which forces compressing vagina and urethra act in keeping continence: those structures have to be repaired during surgical procedures. The Author starts from the wellknown fact that continence is assured by a pressure gradient, cervico-urethral pressure a little greater than bladder pressure; this is due to tone and contraction propriety of smooth and striated urethral musculature, to visceral turgidity consisting in vascular network and to the elastic characteristics of urethral tube; those characteristics are variable from fellow to fellow according with age, hormonal state, previous deliveries, scars or tissue lesions. The sphincteric complex is sufficient to keep continence also for little abdominal pressure increasing; for bigger increasing instead the extra-urethral factors contribution is necessary in maintaining the pressure gradient, that means urethral pressure a little higher than bladder pressure (closure pressure), and continence. Those extra-urethral factors of under stress continence, in the Author view, are due to compression of the urethra on strong structures not permanently acting, but only when the abdominal pressure (stress) causes the physiologic urethro-vesical sphincteric complex displacement below and anteriorly; this displacement stretches the endopelvic fascia that thanks to the different elasticity and resistance of its parts makes different compressive effects on pelvic organs; the zone just below the proximal urethra is the less elastic and the more resistant; thus on this one the compressive force to keep the continence acts. According with this point of view below the proximal urethra and vagina such an hammock-like connectival mass would be present, made by pubo-genito-urinary ligament and sub-urethral connective tissue; the latter is the pubo-cervical fascia, which joints cervix and vagina to pubis and tendineous arch; also the perineal body (mid perineal fascia) and the tendineous perineal center could be part of sub-urethral support non permanently acting.

Connections among continence, posture and body functions apparently independent from urinary, genital and intestinal function are very interesting; at this regard Barbic (43), studying the relation between cough and continence in a group of normally continent women, trough point isolated monopolar electrodes inside levator ani, proved that this muscle contraction precedes the abdominal muscles activation involved in coughing reflex; so levator ani contraction supports and stabilizes bladder neck and lets effective transmission of abdominal pressure on proximal urethra. Deffieux and al. (44, 45), trough electromyographic research, assessed that in continent women the pelvic muscles contraction precedes the abdominal increased pressure due to external intercostal muscles and abdominal muscles contraction during cough.

\section{Defecation}

For what is concerning the pelvic floor musculature, defecation happens for partially voluntary and partially reflex action of both smooth and striated sphincters and pubo-rectal muscle relaxation; so the recto-anal angle opens and consequently feces pass down and are ejected, being not any more blocked by sphincteric tone and contraction. About pubo-rectal muscle, as already said, there are Authors that consider it not as part of levator ani, but a muscle completely independent from the pubo-coccygeus bundle of levator; thus the pubo-rectalis for its connections with the longitudinal intrinsic bundle of rectum and with the striated sphincter, in the Authors' view, would be part of the sphincter complex (33).

The conscious and voluntary part of defecation is referred both to the perception of moment and place socially suitable (that seems to be controlled by cingulum gyre.and internal part of prefrontal zones) and to the voluntary Valsalva action (abdominal straining) that increasing abdominal pressure gets pelvic floor lowering and stimulates the recto-anal inhibitory reflex; this reflex consists in the inhibition of anal sphincters tone (and contraction) and in the inhibition of ano-rectal compression from pubo-rectal muscle. The reflex is greatly dependent from autonomous nervous system, although influences coming from nitric oxide, vasoactive intestinal peptide (VIP) and ATP are also present; the defecatory reflex is regulated by parasympathetic system, while internal sphincter tone and 
the consequent gastroenteric reflex are regulated by orthosympathetic system; the gastroenteric reflex consists in the peristalsis inhibition caused by sphinteric hypertone or contraction; those spinal and superspinal reflexes are caused by the ampulla filling sensation from fecal material pushed down form colic peristalsis (47).

In the defecation physiology it is to be remembered that there are other important reflexes such the colic one, which gives the so called rectal detrusor inhibition and those of bladder reflex afferences: the lowering of bladder sensitivity causes the increasing of filling threshold is the explanation of the fact that micturition and defecation do not happen simultaneously (47).

\section{CoItus}

In man the pelvic floor musculature takes part to this function by pelvic diaphragm contraction according with variability of movements; bulbo-cavernous muscle contraction (mainly of its internal part, so called Houton muscle which like a loop envraps the deep dorsal penile vein) stops the blood emptying contributing to maintain the erection; also ischio-cavernous muscle seems to take part to such a mechanism because its contraction crushes veins but not arteries; (physiologists at this regard seem not to agree). The meanly flat part of the levator ani contributes to a suitable erection through its contraction by which prostate and urethra are lift and the urethral curvature eliminated with consequent urethral and penile straitening. The activity of pubo-rectal bundles that insert on prostate and embrace the seminal vesicles in full coordination with those organs intrinsic contraction helps the ejaculation at least for the emission step. Well-timed reflexes rule urethral and anal sphincters contraction in keeping their closure and inhibiting the just possible micturition and defecation desire. The sudden urethral sphincter relaxation during the emissive ejaculation step seems to cause such a seminal liquid aspiration toward prostatic urethra so creating a bolus ready to be ejected thanks to intrinsic urethral musculature and bulbo-cavernous activity.

In addittion to all that the entire musculo-fascial pelvic apparatus will be strongly activated. in the many times mentioned antagonisms and synergisms together with varies muscular body groups implicated in coital and respiratory movements (48).

Pelvic floor musculature in women. acts in maintaining vaginal introitus open thanks to pubococcygeus fibers called pubo-vaginals in coordination with the collagenelastic-muscular connective tissue which joints pubis (pubo-genita-sacral ligament) and fascia pelvica.

The vaginal modifications due to levator and to connective tissue that fills the anterior part of pelvic hiatus, so called hiatal ligament, are particularly interesting; those structures, pulling laterally from each side, amplify vaginal cavity creating the so called ballooning, suitable in accepting the ejaculate and following ejection in cervical womb, thanks to the pubo-rectal contraction; this muscles activity synchronizes with the intrinsic vaginal muscles activity in creating vaginal volume adaptation to penile dimension, that seems to help in maintaining a full erection. Also in women a lot of sophisticated reflex- es rules vaginal, urethral and anal contraction and relaxation (32).

\section{Vertical position and posture}

The pelvic floor contribution is very important in keeping upright position and posture; this one means the capacity in keeping stable the body position, still or in motion, in balance with gravity center falling on the support base (between the two feet in upright position). Posture is an active, reflex and involuntary phenomenon caused by tone, contraction and relaxation of muscles which set themselves against the gravity force (anti-gravitary or postural muscles); vestibular afferences inform cerebellum which in its turn constantly informs all the body of where could be its gravity center (12).

For what is concerning the upright position. the gluteous major big development (compared to other hominids without constant and obliged up right position) is to be considered; synergism and antagonism with lumbar and abdominal muscles (particularly oblique and transverse) are constant also if in different kind of tone and contraction type; the pelvic musculature acquired indeed a specific characteristics regarding tone, automatism and voluntary, or at least conscious, contraction; pelvic diaphragm muscles tone consists, as already said, in an intermediate state between tone and contraction useful for the function of support; peculiar and sophisticated neurological central controls ensure the absence of muscular exhaustion otherwise present in contracted fibers; it must be added the solidarity of fascial structures, whose composition includes a lot of smooth muscular tissue, which jointly with striated musculature ensure the suitable support tension.

The contraction, also being conscious, cannot be considered voluntary. at least for the majority of non-visceral bundles fibers; it corresponds to automatism in common with all postural structures answering to a series of intricate stimulations perceived through different kind of .sensible, sensorial, hormonal receptors and to not less complicated reflexes, that rule contraction and relaxation as the occasion may require. Pelvic diaphragm muscles and fasciae represent a functional unity and so they contract simultaneously, bilaterally and synergically, in spite of the fact that it is possible that in certain conditions some groups of fibers could be activated separately (49). Neuroscience recently showed the sophisicated complexity of neuronal connections regarding the reflex correlations between pelvic floor activity and other functions; special synergisms exist with gluteous major (very active in creating and keeping the upright position and coccyx in flexo-extension) and with respiratory muscles, especially the outer intercostals; for what is concerning deep breath and cough it is demonstrated, as said, that pelvic muscles contraction precedes the cough hit (46). This cortical control of synergism is not yet well understood; recently research with Magnetic Functional Resonance has showed an activation of the pre-central encephalic gyrus medial wall for striated voluntary musculature of pelvic diaphragm during gluteous major contraction; such activation doesn't happen during other movements, like a finger flexion. Special receptors for sensitivity and in women for estrogens in pelvic floor muscles have 
essential functions for the peculiar trophism and for automatism progressively decreasing with aging (51-52).

\section{Deambulation}

Pelvic musculature activity during deambulation is noticeable; this musculo-aponeurotic framework has a synergic and antagonistic function with that one of trunk and inferior limbs resulting essential in stabilizing the pelvic ring, stressed by continuous postural movements action; strong spurs are curried out during deambulation on pelvic bones which tend to displace them while on contrary they must be kept fix since their mobility is null (51). The foot walking on the ground urges femur, cotyle and iliac bone of the side to get up; in mean time the suspended limb urge pubis and iliac bone of the side to get down in contemporary sacrum tendency to anteriorize or posteriorize according to monopedal load. Evidently pelvic musculature, synergically with extrapelvic one and with those of trunk must act opposing to those forces and keeping stable the pelvis (4).

The examination of musculo-fascial phenomena in each movement could be too long and any way not pertinent to the aim of this work. It is only convenient to specify that the central neurologic control of the coordination among varies muscular compartments is as ever complex and not completely clarified; it is not to be neglected how the muscular tension of pelvic floor urged from walking movements influences statics and dynamics of pelvic organs, particularly urethra and rectum.

\section{Conclusions}

Last years achievements succeeded in clarifying many aspects of pelvic floor activity, whose musculature has been investigated through electromyography, sonography, magnetic resonance, histology, histochemistry, and molecular research. Utilizing recent research concerning not only urinary and gynecologic aspects but also that one regarding statics and dynamics of pelvis and its floor, it is now possible to study this important body part as a unit; that means to consider it in the whole body economy including maintaining upright position, walking, behavior or physical conduct in adjunct to urinary, genital, and intestinal functions.

For more than a century nearly every paper on pelvic floor cited the famous R.J. Dickinson sentence "There is no considerable muscle in the body whose from form and function are more difficult to understand than those of the levator ani, and about which such nebulous impressions prevail" (22); it is today possible to keep the sentence as an historical fact; and consider the pelvic floor as a musculofascial unity, functioning as a unit in synergic and antagonistic activity of muscular bundles, among them more or less interlaced, appraised for their multiple functions and not only for the pelvic cup closure. It is then possible to hope that the above named contradictions (19) are going towards their elimination.

\section{ACKNOWLEDGEMENTS}

An Italian version of the paper with illustrations is available on www.siud.it.

\section{REFERENCES}

1. Sherrington $C$, cited by Tobias in reference 2 .

2. Tobias Ph.V. Il bipede barcollante: corpo, cervello, evoluzione umana. Einaudi, Torino, 1992, p. 821.

3. Traetta J. Gymnastic basic paperback. 1979.

4. Passigli S. Fisiologia articolare del cingolo pelvico. Anatomia, 2008.

5. Rocca Rossetti S. Diaframma. Enciclopedia Medica S.E.S, Sansoni Editore, 1950, vol. IV, p. 750.

6. Virno V. I Sistemi digastrici del diaframma umano, Ricerche di morfologia. Roma, 1925.

7. Virno V. La morphologie du canal aortico-diaphrammatique chez l'homme. Comptes Rendues de l'Association des Anatomistes. 1925, p. 384.

8. Wallner C. Development of the pelvic floor: implications for clinical anatomy, PhD thesis. Faculty of Medicine (AMC-UvA), 2008.

9. Wallner C, van Wissen J, Maas CP, et al. The contribution of levator ani nerve and the pudendal nerve to the innervation of the levator ani muscles; a study in human fetuses. Eur Urol. 2008; 54:1136-42.

10. Cherry DA, Rothenberger DA. Pelvic floor physiology. Surg Clin North Am. 1988; 68:1217-30.

11. Wallner C. Is the puborectalis muscle part of the levator ani muscle? Dis Colon Rectum. 2008; 51:1165-6.

12. Sala F, Tramontano V, Squintani G, et al. Neurophysiology of complex spinal cord untethering.J Clin Neurophysiol. 2014; 31:326-36.

13. Stafford RE, Ashton-Miller JA, Sapsford R, Hodges PW. Activation of the striated urethral sphincter to maintain continence during dynamic tasks in healthy men. Neurourol Urodyn. 2012; 31:36-43.

14. Moschi A., Monticone M. Biomeccanica della articolazione sacro-iliaca, Atti Primo Convegno della Sezione SIMFER di Riabilitazione Ambulatoriale. Pavia, 22 Nov 2003.

\section{Clippinger KS. Human Kinetics. 2007; p.533.}

16. Morren GL, Beets-Tan RG, van Engelshoven JM. Anatomy of the anal canal and perianal structures as defined by phased-array magnetic resonance imaging. Br J Surg. 2001; 88:1506-1512.

17. Schmeiser G, Putz R. The anatomy and function of the pelvic floor. Radiologe. 2000; 40:429-36.

18. Messelink B, Benson T, Berghmans B, et al. Standardization of terminology of pelvic floor muscle function and dysfunction: Report from the Pelvic Floor Clinical Assessment Group of the International Continence Society Neurourol Urodyn. 2005; 24:374-80.

19. Kearney R, Sawhney R, DeLancey JO. Levator ani muscle anatomy evaluated by origin-insertion pairs. Obstet Gynecol. 2004; 104:168-73.

20. Bharucha AE. Pelvic floor: anatomy and function. Neurogastroenterol Motil. 2006; 18:507-519.

21. Fröhlich B, Hötzinger H, Fritsch H. Tomographical anatomy of the pelvis, pelvic floor, and related structures. Clin Anat. 1997; 10:223-30.

22. Dickinson RJ. Studies of levator ani muscle 1889, cited in Dickinson RJ. The time-element in saving the perineum. NY Med J. 1890; 51:371-4.

23. Fritsch H, Lienemann A, Brenner E, Ludwikowski B. linical anatomy of the pelvic floor. Adv Anat Embryol Cell Biol. 2004; 175:III-IX, 1-64. 
24. Frea B. Il pavimento pelvico maschile: cingolo osseo, muscoli, connettivo e innervazione. Ottavo Corso di Urologia Funzionale, Torino, 10-11 Ott. 2014.

25. Cervigni M. Il pavimento pelvico femminile: cingolo osseo, muscoli, connettivo e innervazione. Ottavo Corso di Urologia Funzionale, Torino, 10-11 Ott. 2014.

26. Meschia M. Pavimento Pelvico e Dintorni. Ottavo Corso di Urologia Funzionale, Torino, 10-11 Ott. 2014.

27. Vercelli A. Pavimento Pelvico e Dintorni. Ottavo Corso di Urologia Funzionale, Torino, 10-11 Ott. 2014.

28. Barber MD. Contemporary views on female pelvic anatomy. Cleve Clin J Med. 2005; 72(Suppl 4):S3-11.

29. Singh K, Jakab M, Reid W, et al. Three-dimensional magnetic resonance imaging assessment of levator ani morphologic features in different grades of prolapse. Am J Obstet Gynecol. 2003; 188:910-15.

30. Crapp AAR, Cuthbertson AM. William Waldeyer and the rectosacral fascia. Surg Gynecol Obstet. 1974; 138:252-56.

31. Ayoub SF. The anterior fibres of the levator ani muscle in man. $J$ Anat. 1979; 128:571-80.

32. Robertson DG, Fleming D. Kinetics of standing broad and vertical jumping Can J Sport Sci. 1987; 12:19-23.

33. Shafik A. The role of the levator ani muscle in evacuation, sexual performance and pelvic floor disorders. Int Urogynecol J Pelvic Floor Dysfunct. 2000; 11:361-76.

34. Carbone G, Rocca Rossetti S. Fisiopatologia della vescica neurogena. Relazione al XLI Congresso della Società Italiana di Urologia, Fiuggi, 1968.

35. de Groat WC, Yoshimura N Mechanisms underlying the recovery of lower urinary tract function following spinal cord injury. Prog Brain Res. 2006; 152:59-84.

36. Rocca Rossetti S. La ritenzione urinaria nell'anziano. Fisiopatologia, clinica, terapie. G Gerontol. 1978; 34:734-5.

37. Rocca Rossetti S, Falqui V, Giordano D, et al. Il problema pielectasia pieloplastica interpretato alla luce dell'idrostatica Boll. Atti Soc Urol Centro Merid. Isole 1969-70; 5:1868.

38. Ashton-Miller J, DeLancey JOL. Functional anatomy of the female pelvic floor Ann. N.Y. Acad Sci. 2007; 1101:266-296.

39. Sasaki H, Hinata N, Kurokawa T, Murakami G. Supportive tissues of the vagina with special reference to a fibrous skeleton in the perineum: A review. OJOG Open Journal of Obstetrics and Gynecology. 2014; 4:144-157.

40. Lazarevski MB. Biomechanics of urinary stress incontinence surgery: Theory of the non-permanently acting suburethral supportive structure. Int Urogynecol J Pelvic Floor Dysfunct. 2000; 11:377-85.

41. Carone R. La teoria uretro-centrica. Litografia Saba S.r.l., Roma maggio 2011.

42. Enhorning G. Simultaneous recording of intravesical and intraurethral pressure. A study on urethral closure in normal and stress incontinent women. Acta Chir Scand Suppl. 1961; (Suppl 276):1-68.

43. Barbic M, Kralj B, Cor A. Compliance of the bladder neck supporting structures: importance of activity pattern of levator ani muscle and content of elastic fibers of endopelvic fascia. Neurourol Urodyn. 2003; 22:269-76.

44. Deffieux X, Raibaut P, Rene-Corail P, et al. External anal sphincter contraction during coug: not a simple spinal reflex. Neurourol Urodyn. 2006; 25:782-787.

45. Deffieux X, Hubeaux K, Porcher R, et al. External intercostal muscles and external anal sphincter electromyographic activity during coughing. Int Urogynecol J Pelvic Floor Dysfunct. 2008; 19:521-524.

46. Stebbing JF, Brading AF, Mortensen NJ. Nitric oxide and the rectoanal inhibitory reflex: retrograde neuronal tracing reveals a descending nitrergic rectoanal pathway in a guinea-pig model. $\mathrm{Br} \mathrm{J}$ Surg. 1996; 83:493-8.

47. Kunze WA.Furness JB. The enteric nervous system and regulation of intestinal motility. Annu Rev Physiol. 1999; 61:117-42.

48. Floyd K, McMahon SB, Morrison JFB. Inhibitory interaction between colonic and vescical afferents in the micturition reflex of the cat. J Physiol. 1982; 322:45-52.

49. Hodges PW, Sapsford R, Pengel LHM. Postural and respiratory functions of the pelvic floor muscles. Neururol Urodyn. 2007; 26:362-71.

50. Kenton E, Bubaker R. Relationship between levator ani contraction and motor unit activation in the urethral sphincter. J Obstet Gynecol. 2002; 187:403-6.

51. Bernstein IT. The pelvic floor uuascles: muscle thickness in healthy and in urinary incontinent women measured by ultrasonography with reference to the effect of pelvic floor training. Estrogen receptor studies. Neurourol Urodyn. 1997; 16:237-75.

52. Dimitriou M. Human muscles spindle sensitivity reflects the balance of activity between antagonistic muscles. J Neurosc. 2014; 34:13644-55.

\section{Correspondence}

Salvatore Rocca Rossetti, MD (Corresponding Author)

salvatore.roccarossetti@unito.it

Professor of Urology, University of Torino, Torino, Italy 